On the Self-induction of Solenoids of Appreciable Winding Depths

This content has been downloaded from IOPscience. Please scroll down to see the full text. 1914 Proc. Phys. Soc. London 27371

(http://iopscience.iop.org/1478-7814/27/1/328)

View the table of contents for this issue, or go to the journal homepage for more

Download details:

IP Address: 131.111.164.128

This content was downloaded on 25/08/2015 at 15:49

Please note that terms and conditions apply. 
XXVIII. On the Self-induction of Solenoids of Appreciable Winding Depths. By S. ButTeRworth, M.Sc., Lecturer in Physics, School of Technology, Manchester.

RECEIVED FEBRUARY 27, 1915.

1.

THE only formulæ which appear to have been given for the self-induction of solenoids in which the correction for winding depth is included are those of Rosa* and Cohen. $f$ In arriving at these formulæ certain doubtful approximations have been made. In the present Paper formulæ have been developed which are free from such approximations, ++ and it is shown that, while Rosa's formula gives better results than that of Cohen, it does not possess the accuracy claimed for it by its author.

\section{Cohen's Method.}

The coil under consideration is divided into a finite number of layers, each of which is sufficiently thin to be treated as a cylindrical current sheet. 'The self-induction of the coil is then found by combining the self and mutual inductions of these lavers according to the usual laws. For the self-inductions Cohen uses the formula,

$$
\mathrm{L}=-4 \pi^{2} n^{2} a^{3}\left(\frac{2+c^{2}}{\sqrt{4+c^{2}}}-\frac{8}{3 \pi}\right), \ldots . . . \quad .
$$

in which $a$ is the mean radius of a layer, $c a$ the coil length, and $n$ the number of turns per unit length.

The formula is an approximation to an exact elliptic integral formula due to Lorenz.

For the mutual inductions he uses

$$
\mathrm{M}=4 \pi^{2} n^{2} a^{3}\left(\sqrt{c^{2}+a^{2} / \mathrm{A}^{2}}-\mathrm{A} / a+a / 8 \mathrm{~A}\right), \quad \text {. . . }
$$

in which $a$ and $\mathbf{A}$ are the radii of the inner and outer cylinder

* Rosa, "Bulletin " of the Bureau of Standards, 4, p. 369, 1908.

† Cohen, "Bulletin " of the Bureau of Standards, 4, p. 384, 1908.

\$The formulæ of this Paper are approximate in that they negleot $(a)$ the insulation space between the wires of the coil, $(b)$ the effect of the helioity of the winding.

VOL. XXVII. 
respectively, and $c a$ is the length of the cylinders. This is an approximation to a formula due to Maxwell.*

The final formula obtained by Cohen for the thick coil is

$$
\begin{aligned}
\mathrm{L} / \pi^{2} \mathrm{~N}^{2} \mathrm{R}^{3} & =\frac{4}{m}\left(\frac{2+c^{2}}{\sqrt{4}+c^{2}}-\frac{8}{3 \pi}\right) \\
& +\frac{8}{m^{2}}\left[\left\{(m-1) k_{1}{ }^{2}+(m-2) k_{2}{ }^{2}+\ldots\right\}\left(\sqrt{k_{1}{ }^{2}+c^{2}}-\frac{7}{8} k_{1}\right)\right. \\
& -\frac{\delta k}{2}\left\{m(m-1) k_{1}{ }^{2}+(m-1)(m-2) k_{2}{ }^{2}+\ldots\right\}\left(1-\frac{k_{1}}{\sqrt{k_{1}{ }^{2}+c^{2}}}\right) \\
& \left.-\frac{\delta k}{16}\left\{m(m-1) k_{1}{ }^{2}+(m-2)(m-3) k_{2}{ }^{2}+\ldots\right\}\right], \ldots . \quad \text {. (3) }
\end{aligned}
$$

in which $m$ is the number of layers, $N$ the number of turns per unit length (including all the layers), $c \mathrm{R}$ the coil length, $k_{1} \mathrm{R}$, $k_{2} \mathrm{R}, \ldots$ the mean radii of the layers $\left(k_{r+1}>k_{r}\right)$, and $\mathrm{R} \delta k$ the width of each layer.

- He states that this formula has a minimum accuracy of onehalf of 1 per cent. when $c>4$.

An a priori test of this assertion may be obtained by an examination of formulæ (1) and (2). Since the distance apart of contiguous layers is small compared with their radii, (2) should ayproximately agree with (1) when $\mathrm{A}=a$, i.e.,

$$
\mathrm{I}=4 \pi^{2} N^{2} a^{3}\left(\sqrt{1+c^{2}}-\frac{7}{8}\right) . \quad . \quad . \quad . \quad . \quad . \quad .
$$

should not differ by more than one-half of 1 per cent. from (1) when $c>4$.

Further, we may test the absolute accuracy of (1) and (2) by means of some exact formula, such as that of Havelock, $\uparrow$ viz.,

$$
\left.\left.\left.\mathrm{L}=4 \pi^{2} \mathrm{~N}^{2} a^{2} c\left\{1-\frac{8}{3 \pi c}+\frac{1}{2 c^{2}}\right)-\frac{1 \cdot 3}{2 \cdot 3} \frac{1}{c^{2}}\right)-\frac{3 \cdot 5}{3 \cdot 4} \frac{1}{c^{2}}\right) \ldots\right\} .
$$

in which the notation ) $x$ means that any term is obtained from the preceding term by multiplying by $x_{\ddagger}^{+}$

* Maxwell, "Electricity and Magnetism" (Vol. II., Art. 678).

† Havelock, "Phil. Mag.," Vol. XV., p. 332, 1908.

¥ By using this notation, computation from the formula is far more rapid than when each term in the series is calculated separately. 
Table I. gives the values of $L / 4 \pi^{2} N^{2} a^{3}$ as obtained from the three formulæ.

TABLE I.

\begin{tabular}{|c|c|c|c|}
\hline c. & Havelock, & Formula (1). & Formula (4). \\
\hline $\begin{array}{ccc}4 & \ldots & \ldots \\
5 & \ldots & \ldots \\
6 & \ldots \ldots \ldots\end{array}$ & $\begin{array}{l}3 \cdot 2725430 \\
4 \cdot 2492672 \\
5 \cdot 2333879\end{array}$ & $\begin{array}{l}3 \cdot 1761 \\
4 \cdot 1650 \\
5 \cdot 1596\end{array}$ & $\begin{array}{l}3 \cdot 2481 \\
4 \cdot 2240 \\
5 \cdot 2078\end{array}$ \\
\hline
\end{tabular}

The values from Havelock's formula are calculated to eight figures as they are needed later. The table shows that neither of the fundamental formula used by Cohen are sufficiently accurate to give results to one-half of 1 per cent.

\section{Rosa's Method.}

The inductance $\left(\mathrm{L}_{0}\right)$ of a cylindrical current sheet of the mean radius of the thick coil is calculated by any suitable exact formula, and the correction for thickness is got by deducting from $\mathrm{L}_{0}$ an amount $\Delta \mathrm{L}$, where

$$
\frac{\Delta \mathrm{I}}{\pi^{2} \mathrm{~N}^{2} \mathrm{R}^{3}}=\frac{8}{\pi} c \mathrm{~T}(\mathrm{~A}+\mathrm{B}), \quad . \quad . \quad . \quad . \quad .
$$

in which $\mathrm{N}, \mathrm{R}, c$ have the same meanings as before, $2 \mathrm{TR}$ is the winding depth, and $A$ and $B$ are tabulated functions of $T$ and $c / T$ respectively. The following values of $A$ and $B$ taken from Rosa's tables are required later :--

TABLE II.

\begin{tabular}{|c|c|c|c|}
\hline$c$. & T. & A. & B. \\
\hline $4 \ldots \ldots \ldots$. & $1 / 10$ & 0.6922 & 0.3099 \\
$5 \ldots \ldots \ldots$. & $1 / 12$ & 0.6926 & 0.3218 \\
$6 \ldots \ldots \ldots$ & $1 / 10$ & 0.6922 & 0.3218 \\
\hline
\end{tabular}

The method of obtaining $A$ and $B$ is to divide the coil into slices by planes perpendicular to the axis, such that each slice forms a coil with a winding channel of square section. The correction $\mathbf{A}$ arises from the difference between the selfinduction of a coil of square section and that of a current sheet of the mean radius of the coil. Weinstein's formula is used for the square-sectioned coil and Rayleigh's formula for the current sheet. Apart from the question of the validity of Weinstein's formula up to $2 \mathrm{~T}=0.25$ (the maximum thickness for which Rosa tabulates $A$ ), there appears to be no serious error in this correction.

The correction $B$ is due to the mutual induction between the slices. It is obtained by the method of geometrical mean. 
distance, and herein lies the most probable source of error, as the method of geometrical mean distance is only valid tor coils whose sectional dimensions and distance apart are small compared with their mean radii. The latter condition is far from being the case with coils of the nature under consideration. It will be shown in Section 10 that Rosa's correction is 2 per cent. in error for coils whose length is four times their radius, while the error in the total induction is 0.2 per cent.

\section{Present Method.}

The coil is first taken to be a portion of an infinite coil, and the self-induction $\left(\mathrm{L}_{1}\right)$ calculated under these conditions. The work to be done to remove the portion from the remainder of the infinite coil is then determined, and this gives the diminution in self-induction due to the effect of the pole faces of the coil. This work is due to the attraction of two pairs of unlike poles in contact, together with the repulsion of two pairs of like poles at a distance apart equal to the length of the coil, or, since the work to be done to separate a pair of poles is equivalent to the mutual induction between the corresponding semi-infinite coils, the end correction to be deducted from $\mathrm{I}_{1}$ is

$$
2 \mathrm{M}(o)-2 \mathrm{M}(l) \text {, }
$$

where $\mathbf{M}(l)$ represents the mutual induction between two semi-infinite coils at a distance, $l$.

Hence, if $\Delta \mathrm{L}_{1}$ represents the (additive) thickness correction for $\mathrm{I}_{1}, \Delta \mathrm{M}(l)$ that for $\mathrm{M}(l)$, the total thickness correction is

$$
\begin{array}{r}
\Delta \mathrm{L}=\Delta \mathrm{L}_{1}-2 \Delta \mathrm{M}(0)+2 \Delta \mathrm{M}(l) \\
\text { 5. The Correction } \Delta \mathrm{L}_{1} .
\end{array}
$$

The self-induction of a length, $l$, of an infinite coil of outer and inner radii $a$ and $b$ is given by Maxwell* as

$$
\mathrm{L}_{1}=-\frac{2}{3} \pi^{2} \mathrm{~N}^{2} l\left(a^{2}+2 a b+b^{2}\right),
$$

where $\mathbf{N}$ is the number of turns per unit length. Hence, if $a=\mathrm{R}(1+\mathrm{T}), b=\mathrm{R}(1-\mathrm{T}), l=\mathrm{R} c$

$$
\mathrm{L}_{1}=-1 \pi^{2} \mathrm{~N}^{2} \mathrm{R}^{3} c\left(1-\frac{2}{3} \mathrm{~T}+\frac{1}{3} \mathrm{~T}^{2}\right),
$$

so that the thickness correction $\Delta \mathrm{L}_{1}$ is given by

$$
\frac{\Delta \mathrm{L}_{1}}{\pi^{2} \mathrm{~N}^{2} \mathrm{R}^{3}}=-\frac{8}{3} c \mathrm{~T}\left(1-\frac{1}{2} \mathrm{~T}\right) \quad . \quad . \quad . \quad . \quad \text {. }
$$

* Maxwell, "Electricity and Magnetism," Vol. II.,"Art. 679. 


\section{The Correction $\Delta \mathrm{M}(o)$.}

The mutual induction between two semi-infinite, coaxial, cylindrical current sheets with coplanar ends is

$$
m=\frac{8}{3} \pi a\left\{\left(a^{2}+b^{2}\right) \mathrm{E}-\left(a^{2}-b^{2}\right) \mathrm{K}\right\} \quad . \quad . \quad .
$$

where $a$ and $b$ are the radii of the two sheets $(a>b)$ and $K$ and $\mathrm{E}$ are complete elliptic integrals of the first and second kinds to modulus $b / a$. When $b / a$ is nearly unity, $\mathrm{K}$ and $\mathrm{E}$ are suitably expressed by the series

$$
\begin{aligned}
\mathrm{K}=\log & \frac{4}{k} \\
+ & \frac{1^{2}}{2^{2}} k^{2}\left(\log \frac{4}{k}-\frac{2}{1 \cdot 2}\right) \\
& +\frac{1^{2} \cdot 3^{2}}{2^{2} \cdot 4^{2}} k^{4}\left(\log \frac{4}{k}-\frac{2}{1 \cdot 2}-\frac{2}{3 \cdot 4}\right) \ldots \\
& +\ldots \\
\mathrm{E}=1 & +\frac{1}{2} k^{2}\left(\log \frac{4}{k}-\frac{1}{1 \cdot 2}\right) \\
& +\frac{1^{2}}{2^{2}} \cdot \frac{3}{4} k^{4}\left(\log \frac{4}{k}-\frac{2}{1 \cdot 2}-\frac{1}{3 \cdot 4}\right) \ldots \ldots \\
& +\ldots
\end{aligned}
$$

in which $k^{2}=1-\frac{b^{2}}{a^{2}}$.

If we put $a=r+x, b=r-x$, so that

$$
k^{2}=\frac{4 x r}{(x+r)^{2}}=4 \frac{x}{r}\left(1-2 \frac{x}{r}+3 \frac{x^{2}}{r^{2}}-4 \frac{x^{3}}{r^{3}}+\ldots\right)
$$

(10) becomes, on application of the usual logarithmic and binominal expansions,

$$
\begin{aligned}
\mathrm{K}=\frac{1}{2} \log \frac{4 r}{x}\left(1+\frac{x}{r}+\frac{1}{4} \frac{x^{2}}{r^{2}}+\frac{1}{4} \frac{x^{3}}{r^{3}}+\ldots\right) \\
\quad-\frac{1}{8} \frac{x^{2}}{r^{2}}-\frac{1}{8} \frac{x^{3}}{r^{3}}+\ldots \\
\mathrm{E}=\frac{1}{2} \log \frac{4 r}{x}\left(2 \frac{x}{r}-\frac{x^{2}}{r^{2}}+\frac{3}{2} \cdot \frac{x^{3}}{r^{3}}-\frac{9}{8} \frac{x^{4}}{r^{4}}+\ldots\right) \\
+1-\frac{x}{r}+\frac{3}{4} \frac{x^{2}}{r^{2}}-\frac{x^{3}}{r^{3}}+\frac{153}{192} \frac{x^{4}}{r^{4}}+\ldots .
\end{aligned}
$$


Applying (11) to (9)

$$
\begin{aligned}
m=\frac{16}{3} \pi r^{3}\left\{1+\frac{3}{4} \frac{x^{2}}{r^{2}}\right. & +\frac{3}{64} \frac{x^{4}}{r^{4}}+\ldots \\
& \left.-\frac{3}{2} \log \frac{4 r}{x}\left(\frac{x^{2}}{r^{2}}-\frac{1}{8} \frac{x^{4}}{r^{4}}+\ldots\right)\right\} . . . .
\end{aligned}
$$

In order to find the mutual induction between two semiinfinite coils, $A$ and $B$, in contact, each having mean radius $\mathrm{R}$, winding depth $2 \mathrm{X}$ and $n$ turns per unit area of section, divide the coils into cylindrical filaments of width $\delta l$ and add the mutual inductions of the filaments as follows:-

Take the mutual induction between a filament of radius $r+x$ in coil $A$ and one of radius $r-x$ in coil B. Associate with it the (same) mutual induction between the filament $r-x$ in $\mathbf{A}$ and $r+x$ in $B$. For these two pairs the sum of the mutual inductions is

$$
\delta \mathrm{M}=2 n^{2} m(\delta l)^{2} \text {. . . . . . . }
$$

Let $r$ vary by steps $\delta r=\delta l$ from $r=\mathrm{R}-\mathrm{X}+x$ to $r=\mathrm{R}+\mathrm{X}-x$ The sum of the expressions $\delta \mathrm{M}$ will then give the sum of the mutual inductions of all filaments at a distance $2 x$ apart.

Perform a second summation with $x$ varying by steps $\delta x=\frac{1}{2} \delta l$ from $x=0$ to $x=\mathrm{X}$. This will give the required mutual induction $\mathbf{M}(0)$. Hence

Applying (14) to (12)

$$
\mathrm{M}(0)=4 n^{2} \int_{0}^{\mathrm{X}} d x \int_{\mathbf{R}-\mathrm{X}+x}^{\mathrm{R}+\mathrm{X}-x} m d r \quad . \quad .
$$

$$
\begin{aligned}
\mathrm{M}(0)=\frac{64}{3} n^{2} \pi \mathrm{R}^{3} \mathrm{X}^{2} & \left\{1+\frac{23}{48} \frac{\mathrm{X}^{2}}{\mathrm{R}^{2}}-\frac{1}{1600} \frac{\mathrm{X}^{4}}{\mathrm{R}^{4}}\right. \\
& \left.-\log \frac{4 \mathrm{R}}{\mathrm{X}}\left(\frac{1}{4} \frac{\mathrm{X}^{2}}{\overline{\mathrm{R}}^{2}}-\frac{1}{80} \frac{\mathrm{X}^{4}}{\mathrm{R}^{4}}\right)\right\} . . .
\end{aligned}
$$

or putting $\mathrm{X}=\mathrm{TR}, 2 n \mathrm{X}=\mathrm{N}$, so that $\mathrm{N}$ is the number of turns per unit length in either coil, the correction $\Delta M(o)$ is given by

$$
\frac{\Delta \mathrm{M}(o)}{\pi^{2} \mathrm{~N}^{2} \mathrm{R}^{3}}=-\frac{4}{3 \pi}\left\{\mathrm{T}^{2}\left(\log \frac{4}{\mathrm{~T}}-\frac{23}{12}\right)-\frac{\mathrm{T}^{4}}{20}\left(\log \frac{4}{\mathrm{~T}}-\frac{1}{20}\right)\right\}
$$

7.

In order to see whether the effect of higher powers of $T$ is appreciable, the mutual induction $\mathrm{M}(o)$ will now be found.by 
another method. In a Paper on thick coaxial coils* the author has shown that the mutual induction $\mathrm{N}(0)$ between two semiinfinite coils of outer radii unity and $r$ respectively, of zero inner radius, and with their ends in contact, is given by

$$
\frac{\mathrm{N}(o)}{2 \pi^{2} r^{3}}=\frac{1}{6}\left\{1-\frac{3}{20} r^{2}\left(\log \frac{4}{r}+\frac{9}{20}\right)+3 r^{4} \sigma\right\}, . .
$$

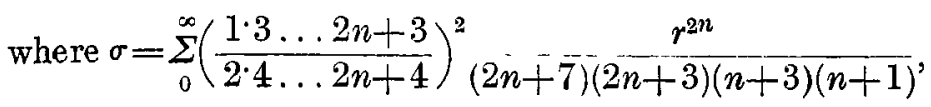

the winding density of each coil being unity. When $r=1$ we have the alternative form

$$
\begin{aligned}
\frac{N_{1}(o)}{2 \pi^{2}} & =\frac{1}{30 \pi}\left\{17-6\left(1-\frac{1}{3^{2}}+\frac{1}{5^{2}}-\frac{1}{7^{2}}+\ldots\right)\right\} \\
& =0.122063419 . . . . . . . . .
\end{aligned}
$$

Further, the mutual induction $\mathbf{M}(o)$ between two similar hollow coils of external and internal radii unity and $r$ is

$$
\mathbf{M}(o)=\left(1+r^{5}\right) \mathrm{N}_{1}(o)-2 \mathrm{~N}(o) . \quad . \quad . \quad .
$$

Since in (17) the general term is given, any desired accuracy may be obtained by calculating a sufficient number of terms, although when $r$ is nearly equal to unity the method becomes tedious, as (19) is then the difference between two nearly equal quantities.

Table III. compares the values of $\mathbf{M}(0)$ as found by (17) and (19), and by (16). The second column is calculated by (17), the third by (19) and the fourth by (16). It is seen that the value of $\mathrm{N}_{1}(o)$ is in good agreement with (18) and that formula (16) is extremely accurate for all winding depths less than onequarter the radius of the coils.

TABLE III.

\begin{tabular}{|c|l|l|l|}
\hline & & \multicolumn{2}{|c|}{$\mathrm{M}(o) / 2 \pi^{2}}$. \\
\hline & $\mathrm{N}(o) / 2 \pi^{2} r^{3}$. & \multicolumn{1}{|c|}{$\mathrm{By}(19)}$. & \multicolumn{1}{|c|}{$\mathrm{By}(16)}$. \\
\hline 1.00 & $0 \cdot 122063423$ & & \\
0.95 & 0.125119075 & $0 \cdot 001965839$ & 0.0019658390 \\
0.90 & 0.12817228 & $0 \cdot 007265462$ & 0.0072654615 \\
0.85 & 0.13120878 & $0 \cdot 01506643$ & 0.015066436 \\
0.80 & 0.13421539 & 0.02462460 & 0.02462459 \\
\hline
\end{tabular}

* Butterworth, “Phil. Mag.,” p. 578, April, 1915. 


\section{The Correction $\Delta \mathrm{M}(l)$.}

It is shown in the Paper referred to in the preceding section that the mutual induction between two semi-infinite coaxial coils of outer radii unity and $r$, inner radius zero and at distance $z$ apart is given by

$$
\begin{aligned}
\mathrm{N}(z, r)=\frac{\pi^{2} r^{3}}{z}\left\{\frac{1}{9}\right. & -\frac{1}{(2 z)^{2}}\left(\frac{1}{15}+\frac{1}{15} r^{2}\right) \\
& +\frac{1}{(2 z)^{4}}\left(\frac{2}{21}+\frac{6}{25} r^{2}+\frac{2}{21} r^{4}\right) \\
& -\frac{1}{(2 z)^{6}}\left(\frac{5}{27}+\frac{6}{7} r^{2}+\frac{6}{7} r^{4}+\frac{5}{27} r^{6}\right) \\
& +\ldots\}
\end{aligned}
$$

the winding density of each coil being unity and $z$ being greater than $2 r$.

From the laws of combination of mutual inductances and from dimensional considerations, the mutual induction between two similar hollow coils of outer and inner radii $a$ and $b$, and at a distance $c$, is

$$
\mathrm{M}(c)=a^{5}\left\{\mathrm{~N}(z, 1)-2 \mathrm{~N}(z, r)+r^{5} \mathrm{~N}\left(\frac{z}{r}, 1\right)\right\}, .
$$

where $z=c / a, r=b / a$.

The relation (21) when applied to (20) converts the term $r^{2 n+3} / z^{2 p+1}$ into $\frac{a^{5}}{z^{2 p+1}}\left(1-2 r^{2 n+3}+r^{2 p+6}\right)$ and the term $r^{2 p-2 n+3} / z^{2 p+1}$ into $\frac{a^{5}}{z^{2 p+1}}\left(1-2 r^{2 p-2 n+3}+r^{2 p+6}\right)$.

Since these terms have the same coefficient their sum is converted into

$$
\begin{gathered}
\frac{2 a^{5}}{z^{2 p+1}}\left(1-r^{2 n+3}\right)\left(1-r^{2 p-2 n+3}\right) \\
\text { or } \quad \frac{2}{c^{2 p+1}}\left(a^{2 n+3}-b^{2 n+3}\right)\left(a^{2 p-2 n+3}-b^{2 p-2 n+3}\right) . \quad \text {. }
\end{gathered}
$$

Hence, if in (20) we replace $2 r^{2 n+3} / z^{2 p+1}$ by the expression (22) we obtain the required series for $M(c)$. Making this 
substitution, and in addition putting $a=1+\mathrm{T}, b=1-\mathrm{T}$, we find on arranging in ascending powers of $T$

$$
\begin{aligned}
\frac{c \mathrm{M}(c)=}{4 \pi^{2}}= & \mathrm{T}^{2}\left(1-\frac{1}{2 c^{2}}+\frac{5}{8 c^{4}}-\frac{35}{32 c^{6}}+\ldots\right) \\
& +\frac{2}{3} \mathrm{~T}^{4}\left(1-\frac{7}{4 c^{2}}+\frac{17}{4 c^{4}}-\frac{775}{64 c^{6}}+\ldots\right) \\
& +\frac{1}{9} \mathrm{~T}^{6}\left(1-\frac{39}{10 c^{2}}+\frac{507}{20 c^{4}}-\frac{2167}{80 c^{6}}+\ldots\right) \\
& +\ldots, \quad . \quad . \quad . \quad . \quad \text {. . . . . . . }
\end{aligned}
$$

which holds for coils of mean radius unity, winding depth $2 \mathrm{~T} \Gamma$, length $c$, and unit winding density. By multiplying $\mathbf{M}(c)$ by $n^{2} R^{3}$ we get the formula for coils of mean radius $R$, winding depth $2 \mathrm{TR}$, length $c \mathrm{R}$, and winding density $n$. Finally, since $2 n \mathrm{TR}=\mathrm{N}$, the number of turns per unit length

$$
\begin{aligned}
\frac{\Delta \mathrm{M}(l)}{\pi^{2} \mathrm{~N}^{2} \mathrm{R}^{3}}= & \frac{2}{3} \frac{\mathrm{T}^{2}}{c}\left(1-\frac{7}{4 c^{2}}+\frac{17}{4 c^{4}}-\frac{775}{64 c^{6}}+\ldots\right) \\
& +\frac{1}{9} \frac{\mathrm{T}^{4}}{c}\left(1-\frac{39}{10 c^{2}}+\ldots\right) \\
& +\ldots . \text {. . . . . . . . . . . }
\end{aligned}
$$

9.

As a check on (24), the value of $\mathrm{M}(l)$ can be calculated by (24) and again by (20) and (21) directly. In getting $\mathbf{M}(l)$ for the mean radius the formula

$$
\left.\left.\left.\mathrm{M}(l)=\frac{\pi^{2} \mathrm{~N}^{2} \mathrm{R}^{3}}{c}\left\{1-\frac{1}{2 c^{2}}\right)-\frac{3 \cdot 5}{3 \cdot 4} \frac{1}{c^{2}}\right)-\frac{5 \cdot 7}{4 \cdot 5} \frac{1}{c^{2}}\right) \ldots\right\}, .
$$

due to Havelock, may be used. The first term in (23) agrees with (25).

For example, with $R=9,2 T R=2, c R=40, N=200$,

(20) and (21) give $M=6 \cdot 454_{3} \pi^{2}$ millihenries.

(24) and (25) give $\mathrm{M}=6.4544_{1} \pi^{2}$ millihenries.

The agreement is satisfactory within the limits of accuracy of the formulæ. 


\section{Comparison of the Corrections.}

Table IV. shows the results obtained by Rosa's, Cohen's. and the present method for three coils, for which

$$
c=4,5,6, ; \mathrm{T}=1 / 10,1 / 12,1 / 10, \pi^{2} \mathrm{~N}^{2} \mathrm{R}^{3}=1 \text {. }
$$

It is seen that Rosa's correction is 2 per cent. too high, while the total induction is one-fifth per cent. low.

In Cohen's method the tabulated values are for five layers. Although the results show very good agreement with the correct values, this is accidental, being due to the number of layers chosen. If a larger number of layers are taken the results get worse ir.stead of better. Thus, for $c=4, T=1 / 10$, with

$$
\begin{aligned}
& m=\begin{array}{lllllll}
1 & 2 & 3 & 4 & 5 & 10 & \text { infinity }
\end{array} \\
& \mathrm{L}=12 \cdot 70,12 \cdot 11,12 \cdot 07,12 \cdot 06,12 \cdot 09,12 \cdot 14,12 \cdot 19
\end{aligned}
$$

\begin{tabular}{|c|c|c|c|}
\hline 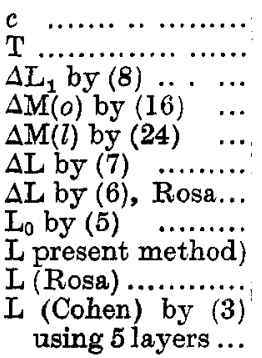 & $\begin{array}{c}4 \\
1 / 10 \\
-1.0133333 \\
-0.0075138 \\
+0.0015093 \\
-0.995287 \\
-1.0207 \\
13.090172 \\
12.094885 \\
12.0695\end{array}$ & $\begin{array}{c}5 \\
1 / 12 \\
-1.0690236 \\
-0.0057568 \\
+0.0008676 \\
-1.055775 \\
-1.0763 \\
16.997069 \\
15.941294 \\
15.9208 \\
15.939\end{array}$ & $\begin{array}{c}6 \\
1 / 10 \\
-1 \cdot 5200000 \\
-0 \cdot 0075138 \\
+0.0010621 \\
-1 \cdot 502848 \\
-1 \cdot 5492 \\
20 \cdot 933552 \\
19 \cdot 430704 \\
19 \cdot 3843 \\
19 \cdot 462\end{array}$ \\
\hline
\end{tabular}

the correct value being $12 \cdot 09$.

\section{TABLE IV.}

11.

It is convenient to summarise the steps by which the inductance of a solenoid can be calculated. The coil is assumed to have a length greater than twice its diameter and a thickness of winding of less than one-tenth the diameter. The formuln are sufficient to give four figure accuracy in the final result. The method is as follows:-

1. Calculate the inductance for the mean radius $R$ without. the end correction trom th: formula,

$$
\mathrm{L}_{1}=4 \pi^{2} \mathrm{~N}^{2} \mathrm{R}^{3} c \text {, }
$$

where $\mathrm{N}$ is the number of turns per unit length (including all layers) and $R c$ is the coil length.

2. Apply the "end" correction to obtain the inductance. 
$\left(L_{2}\right)$ for a current sheet of the mean radius of the coil from the formula,

$$
\mathrm{L}_{2}==\mathrm{L}_{1}\left(1-\frac{8}{3 \pi c}+\frac{1}{2 c^{2}}-\frac{1}{4 c^{4}}\right)
$$

3. Apply the "thickness" correction to obtain the inductance $\left(\mathrm{L}_{3}\right)$ for a coil of winding depth $2 \mathrm{TR}$ from the formula,

in which

$$
\mathrm{L}_{3}=\mathrm{L}_{2}+\Delta \mathrm{L}_{2} \text {, }
$$

$$
\Delta \mathrm{L}_{2}=-\frac{2}{3} \mathrm{~L}_{1} \mathrm{~T}\left\{1-\frac{\mathrm{T}}{2}-\frac{\mathrm{T}}{\pi \mathrm{c}}\left(\log _{0} \frac{4}{\mathrm{~T}}-\frac{23}{12}\right)\right\} .
$$

4. Apply the "insulation space" correction to obtain the tru. 3 inductance L, for which see Rosa ("Bull." Bureau of Standards, 3, p. 37, 1907).

\section{Summary.}

1. It has been shown that the formulæ previously given for correcting for thickness in determining the inductance of solenoids fail to give an accuracy of one part in a thousand.

2 . The true correction formulæ are obtained which are capable of giving eight figure accuracy without undue labour.

3. Simplified formulæ are also given which hold when only four figure accuracy is required.

\section{APPENDIX.}

As portions of the criticism of Rosa's and Cohen's formulæ depend on arithmetical accuracy in calculation, the arithmetical details for $c=4, T=1 / 10$ are appended.

(a) Inductance of Mean Layer Solenoid.

By Havelock's formula (5) :

$$
\begin{aligned}
& \mathrm{L}_{0}=16\{1-0.21220659+0.03125000 \\
& \text { - } \quad 97656+\quad 7630 \\
& \begin{array}{l}
-\quad 835+\quad 109 \\
-\quad 16+
\end{array} \\
& \begin{array}{ll}
-\quad 16+\quad 3\}
\end{array} \\
& =16\{1.03132742 \\
& -0 \cdot 21319166\} \\
& =16 \times 0.81813576 \\
& =13.090172 \text {. }
\end{aligned}
$$

(b) Correction $\Delta \mathrm{I}_{1}$ (formula (8)).

$$
\Delta \mathrm{L}_{1}=-\frac{8}{3} \times 4 \times \frac{1}{10}(1-0.05)=-\frac{30 \cdot 4}{30}=+1.0133333 \text {. }
$$


(c) Correction $\Delta \mathrm{M}(o)$ (formula (16)).

$$
\begin{aligned}
\Delta \mathrm{M}(o)= & -\frac{4}{3 \pi}\{0.01(3.68888-1.91667)-0.000005(3.69-0.05)\} \\
=- & \frac{4}{3 \pi}\{0.0177221-0.0000182\} \\
= & -0.424413 \times 0.0177039 \\
= & -0.00751376 . \\
& (d) \text { Correction } \Delta \mathrm{M}(l) \text { (formula }(24)) . \\
\Delta \mathrm{M}(l)= & \left\{\begin{array}{cr}
0.0016667-0.0001823 \\
+\quad 277- & 49
\end{array}\right\}+\left\{\begin{array}{rr}
0.0000028 \\
= & 0.0015093 . \\
& (e) \text { Correction } \Delta \mathrm{L}(\text { formula }(7)) . \\
\Delta \mathrm{I}= & \Delta \mathrm{L}_{1}-2 \Delta \mathrm{M}(o)+2 \Delta \mathrm{M}(l) \\
= & -1.0133333+0.0150275+0.0030186 \\
= & -0.9952872 .
\end{array}\right.
\end{aligned}
$$

(f) Rosa's Correction (formula (6) and Table II.).

$$
\begin{aligned}
\Delta \mathrm{L} & =-\frac{8}{\pi} \times \frac{4}{10}(0.6922+0.3099) \\
& =-0.31831 \times 3.2 \times 1.0021 \\
& =-1.0207 .
\end{aligned}
$$

Using five layers.

(g) Cohen's Formula (3).

$$
\begin{aligned}
& m=5 \text {. } \\
& \delta k=0 \cdot 04 . \\
& k_{1}=0.92 \quad k_{2}=0.96 \quad k_{3}=1.00 \quad k_{4}=1.04 \\
& k_{1}{ }^{2}=0.8464 \quad k_{2}{ }^{2}=0.9216 \quad k_{3}{ }^{2}=1.0000 \quad k_{4}{ }^{2}=1.0816 \\
& (m-1) k_{1}{ }^{2}=3.3856 \quad m(m-1) k_{1}{ }^{2}=16.9 \quad m(m-1) k_{1}{ }^{2}=16.9 \\
& (m-2) k_{2}^{2}=2.7648 \quad(m-1)(m-2) k_{2}^{2}=11.1 \quad(m-2)(m-3) k_{2}^{2}=5.5 \\
& (m-3) k_{3}^{2}=2.0000 \quad(m-2)(m-3) k_{3}{ }^{2}=6.0 \\
& (m-4) k_{d}^{2}=1.0816 \quad(m-3)(m-4) k_{1}^{2}=2 \cdot 2 \\
& \text { Sum }=\overline{9.2320} \text { Sum }=\overline{36.2} \text { Sum }=\overline{22.4} \\
& \frac{2+c^{2}}{\sqrt{4+c^{2}}}-\frac{8}{3 \pi}=0.9 \sqrt{20}-\frac{8}{3 \pi}=\left\{\begin{array}{r}
4.0249 \\
-0.8488
\end{array}=3.1761\right. \\
& \sqrt{k_{1}^{2}+c^{2}}-\frac{7}{8} k_{1}=\sqrt{16 \cdot 846}-0 \cdot 805=\left\{\begin{array}{r}
4 \cdot 1045 \\
-0.8050
\end{array}=3 \cdot 2995\right. \\
& 1-\frac{k_{1}}{\sqrt{k_{1}^{2}+c^{2}}}=\frac{3 \cdot 1845}{4 \cdot 1045}=0 \cdot 775 \text {. }
\end{aligned}
$$


Substituting in formula (3)

$$
\begin{aligned}
& \mathrm{L}=\frac{4}{5} \times 3 \cdot 1761+\frac{8}{25}(9 \cdot 2320 \times 3.2995 \\
&\quad-0.02 \times 36.2 \times 0.775-0.056) . \\
&=2.5409+0.32(30 \cdot 461-0.617) \\
&=2.5409+9 \cdot 5501=12.0910 .
\end{aligned}
$$

\footnotetext{
ABSTRACT.

The existing formulæ for coils of this type-viz., those of Rosa and Cohen-are shown to be inaccurate, the error amounting to onefifth of 1 per cent. for the best formula when the winding depth is one-tenth the diameter of the coil. For greater winding depths the error is larger. The inaccuracy in Rosa's formula is due to the neglect of curvature in correcting for thickness, while in Cohen's formula the error is due to the approximate method of development.

New formulæ are developed by methods which are free from such approximations, and which apply to any coil for which the length is greater than twice the diameter, and the winding depth is less than one-tenth the diameter. These formulæ are capable of giving eightfigure accuracy.

Simplified formulæ are also given which are suitable when only four-figure accuracy is required.
} 